\title{
COMPACT CORIOLIS MASS FLOW METER/CONTROLLER FOR LOW FLOWS
}

\section{W. Jouwsma}

Bronkhorst Cori-Tech B.V. Nijverheidsstraat 2-6, 7261 AK Ruurlo, The Netherlands Phone: +31 573 458890; Fax: +31 842 292375;

Internet: www.bronkhorst-cori-tech.com E-mail:info@bronkhorst-cori-tech.com

\section{Introduction}

The accurate and fast measurement of small mass flow rates down to $1 \mathrm{~g} / \mathrm{h}$ of fluids is considered an "enabling technology" in various industries. Applications for instruments capable of measuring these low flow rates can be found in e.g. semiconductor, fine-chemical, pilot plants, food \& pharmaceutical industries and liquid dosing systems for micro reactors. Mass flow meters based on the Coriolis effect offer the most direct sensing of the mass flow rate, and for this reason do not need complicated translation or linearization tables to compensate for other physical parameters (e.g. density, temperature, heat capacity, viscosity, etc.) of the medium that they measure. To measure low mass flow rates using the Coriolis principle the flow tube has to be scaled-down. Conventional Coriolis mass flow meters use e.g. magnets or coils attached to the tube for actuating and sensing. When scaling down conventional designs, the tube becomes so small that any attachment will ruin the performance. Therefore another approach is needed.

This article describes some of the design aspects that have contributed to a new compact Coriolis mass flow meter/controller. Also the performance of the final product is shown.

\section{Sensor construction}

The present instrument deals with innovations on several system aspects, together leading to better overall performance. Tube shape, actuation and sensing are optimized for accurate measurement of low flows using the Coriolis principle. These three aspects are discussed in the following sections. A prototype instrument is depicted in figure 1.

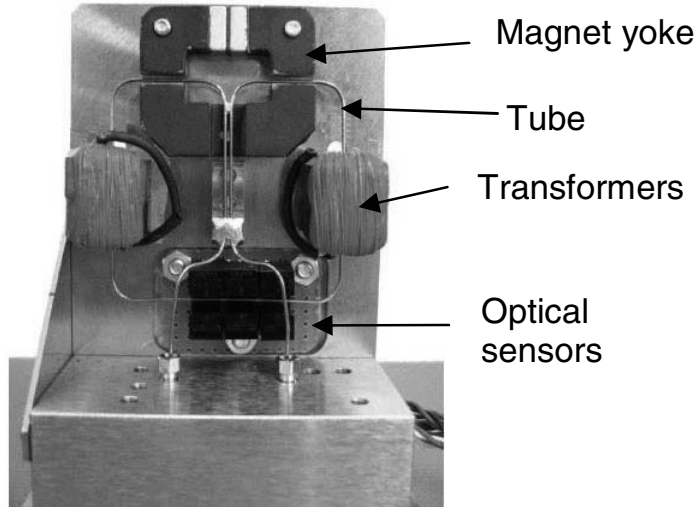

Figure 1a: protoype instrument

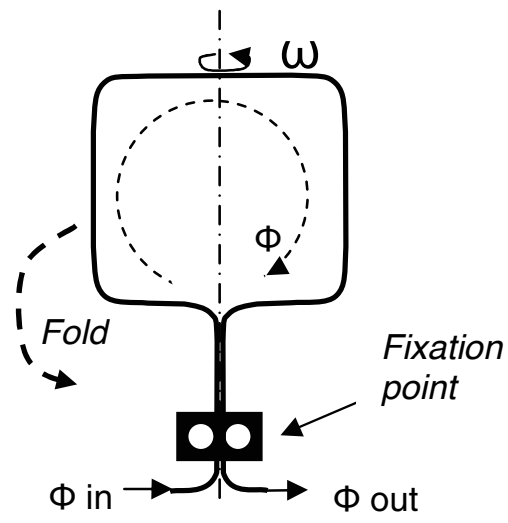

Figure 1b: Principle of tube shape

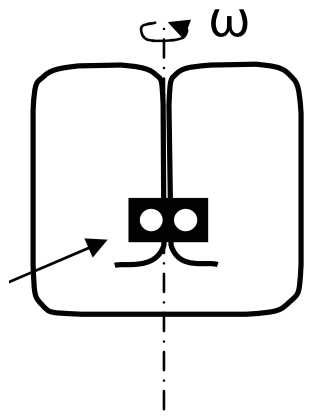

Figure 1c:
Final compact tube shape

\subsection{Tube shape}

The basic principle of the shape of the tube is depicted in figure $1 \mathrm{~b}$. This shape can be folded to the final compact shape as depicted in figure 1c. The loop follows a mechanically closed path, in that the sensing tube has two mutually opposed ends that are connected to a flexible inlet tube and to a flexible outlet tube for the flowing medium, and in that the loop is resiliently suspended from a frame by means of the flexible inlet and outlet tubes such that the resulting suspension allows a movement about two mutually 
perpendicular axes in the plane of the loop, one for the excitation movement and one for the Coriolis movement that arises when a medium is flowing through the tube [1].

The expression 'mechanically closed path' relating to the loop denotes that the loop has an interruption, on either side of which the tube has mutually opposed first and second ends. These ends are mechanically connected to each other. A force can thus be propagated through the loop. It is ensured in this manner that the loop has certain rigidity. Another advantage of a fastening the inlet and outlet tubes next to one another to the frame is that the temperature sensitivity of the flow meter is less than in the case in which the fastening points are away from one another.

The moving tube is attached to the chassis at a single fixation point. Barring the fixation point, there are no attachments upon the tube to the fixed world (e.g. magnets or wires). The sensing tube forms a single loop through which a medium flows during operation.

Excitation means cause the loop to oscillate about an excitation axis of rotation during operation, the axis is in the plane of the loop. When a medium flows through the loop that rotates about the excitation axis, Coriolis forces are generated in the transverse branch and result in a vibration of the loop about an axis perpendicular to the excitation axis of rotation. This vibration, which is proportional to the flow, is superimposed on the fundamental oscillation. Position sensors along the ends of the transverse branch measure the oscillations performed by the excitation and Coriolis force.

\subsection{Actuation}

In conventional systems excitation means used for the excitation of the tube constitute an additional mass to the tube in e.g. magnets or wires. This prevents a change in the excitation frequency as a function of the density of the medium flowing through the tube, with the result that a measurement of the density (an additional property of a Coriolis flow meter) becomes less accurate. The present tube is an intrinsically very light object, and if excitation means are fastened thereto it will cost an additional amount of energy to bring the loop into resonance. It is accordingly preferred to use an excitation technique that does not require the addition of further components to the tube.

The current Coriolis flow meter is characterized in that the excitation means are (electro) magnetic and in operation do not make contact with the tube and have no components that are fastened to the tube. In other words: the excitation means are not connected to the movable portion of the tube.

In this respect, the excitation means comprise means adapted to generate an electric (alternating) current in the wall of the tube, through induction. The electrically conductive tube itself is the secondary turn of a transformer. This principle is clarified in figure 3a. The core of the transformer is positioned around the loop of the tube. For symmetry two identical transformers are placed as depicted in the picture of the prototype (figure 1a).

A permanent magnet generates a magnetic field transverse to the direction of the current in the tube wall so as to exert, through interaction with the current through the tube, electromagnetic forces (so-called Lorentz forces) on the tube with the object of causing the tube to rotate about one of the perpendicular axes [2]. The magnet means comprise a permanently magnetic magnet yoke with two air gaps through which respective tube portions extend. This is clarified in figure $3 \mathrm{~b}$. Torque excitation is used for realizing a twist excitation mode around the $\omega$ axis as depicted in figure 1c. The innovative actuator contributes to a sensitive flow meter with an excitation system that is capable of measuring the medium density more accurately.

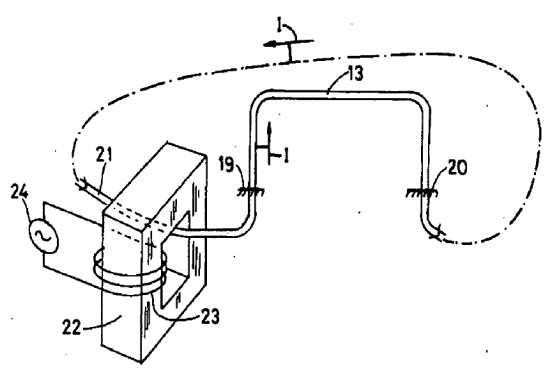

Figure 3a: Current inducer

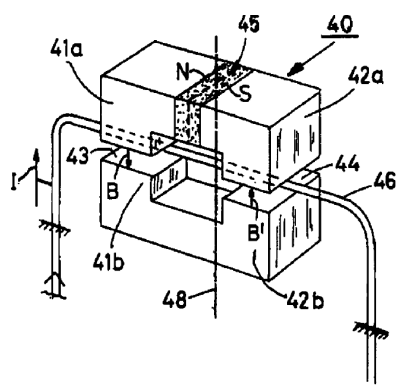

Figure 3b: permanent magnet yoke 


\subsection{Sensing}

The position sensing of the tube is performed by an array of contactless optical sensors [3]. The optical sensors are opto-electronic sensors (fig 4a) which each comprise a light source(116) and a photosensitive cell(117). The light source and the light detector are positioned right opposite to and at a predetermined distance from one another. They are separated by an intervening space in which a vane fastened to the tube can move freely. The light source and the light detector together comprise an optical micro module. It is a U-shaped housing having two legs in one of which a LED and in the other one of which a phototransistor is present. The optical axes of the light source and the light detector are mutually matched, so that the light from the light source directly hits the light detector. The signal generated by the light detector is thus only dependent on displacements of the vane attached to the tube.

The instrument is provided with multiple optical sensors (fig $4 \mathrm{~b}$ ) for measuring deformation of the tube occurring under the influence of a medium flowing through the tube. The sensors are situated on either side of the point of intersection (pole) of the primary axis of rotation and a tube portion of which the deformation is to be measured. Here the measured amplitude of the fundamental oscillation is small while the Coriolis amplitude due to the mass flow is large. Therefore the flow meter has an enhanced sensitivity thanks to the placement of the sensors close to the rotation axis.

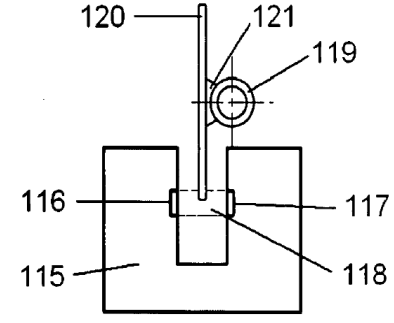

Fig 4a: $\quad$ Cross section of tube with vane positioned in the photo micro sensor

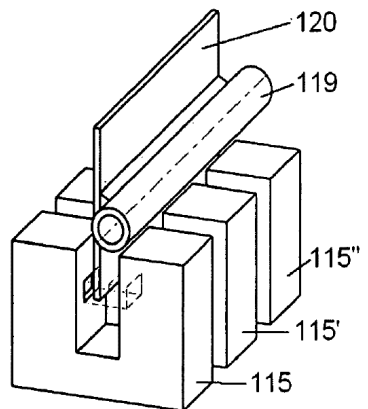

Fig 4b: $\quad$ Tube section with vane in multiple photo micro sensors

With the use of additional electronics for signal conditioning and a DSP (digital signal processor) the ratio between the two oscillation modes can be decomposed from the measured position signals. The ratio between the two oscillations modes (excitation and Coriolis) is a linear measure for the mass flow.

\subsection{The final product}

The final product consists of a range of three instrument types named M12, M13 and M14 with different tube dimensions. These result in three different (nominal) flow ranges. These types and their flow ranges are depicted in table 1 and. Figure 5 shows a picture of the final instrument, the mini Cori-flow. Each tube with its flow range fits inside the same housing. Equipped with an integrated control valve the instrument is also available as a compact flow controller.

\begin{tabular}{|l|l|l|}
\hline \multirow{2}{*}{$\begin{array}{l}\text { Instrument } \\
\text { type }\end{array}$} & \multicolumn{2}{|l|}{ Full scale flow ranges [g/h] } \\
\cline { 2 - 3 } & $\min$ & $\max$ \\
\hline $\mathrm{M} 12$ & $0.5 . .20$ & $0.5 \ldots 500$ \\
\hline $\mathrm{M} 13$ & $2 . .50$ & $2 . .2000$ \\
\hline $\mathrm{M} 14$ & $30 . .1000$ & $30 . .30000$ \\
\hline
\end{tabular}
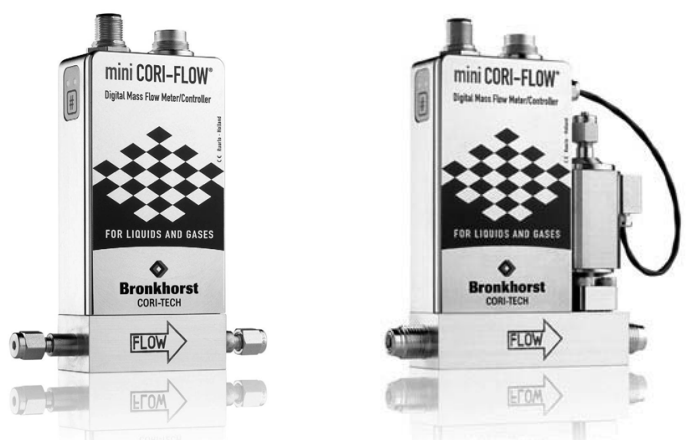

Figure 5 mini CORI-FLOW ${ }^{\circledR}$; the final product meter/controller

\section{Experimental}

Various experiments have been carried out to investigate the performance of the final product. Tests are carried out for determining the accuracy for liquids and gasses. A supply pressure, control valve and the onboard PID controller are used to provide a constant flow through the test devices. A scale is used as a reference for water flow. For measuring the accuracy for gasses a piston prover is used as a reference at 
various flows of $\mathrm{CO} 2$. The test is performed with $\mathrm{CO} 2$ as this is hard to measure accurately with conventional (thermal) mass flow meters. The instruments are calibrated at a certain reference flow to determine the sensitivity. A check has been carried out at multiple points to determine the linearity and accuracy for water and $\mathrm{CO} 2$.

An important issue for the performance of a Coriolis mass flow meter is the stability of the zero (offset) as this limits the accuracy at low flows. The stability of the zero has been tested at different temperatures. This is measured by monitoring the measure value (mass flow) of the instrument at guaranteed zero flow at different temperatures between [0..70] ${ }^{\circ} \mathrm{C}$. The reference temperature is provided to the instruments by placing them in a climate chamber.

The compact instrument is also combined with an electromagnetic control valve to obtain a compact flow controller. An important issue with Coriolis mass flow meters and controllers is the pressure drop. Tests have been carried out to measure the pressure drop over a meter and controller for water and air.

\section{Results \& discussion}

\subsection{Accuracy of flow measurement}

The mass flow accuracy of the instrument with lowest flow range (M12) is depicted below. The excellent zero stability of $0.1 \mathrm{~g} / \mathrm{h}$ of the instrument results in an accuracy of less than $1 \%$ reading at $10 \mathrm{~g} / \mathrm{h}$ for water and $\mathrm{CO} 2$.

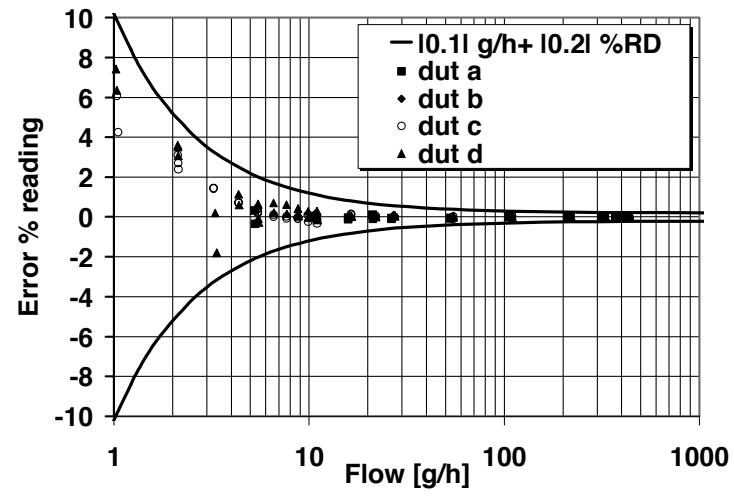

Chart 1a Accuracy of M12 (water)

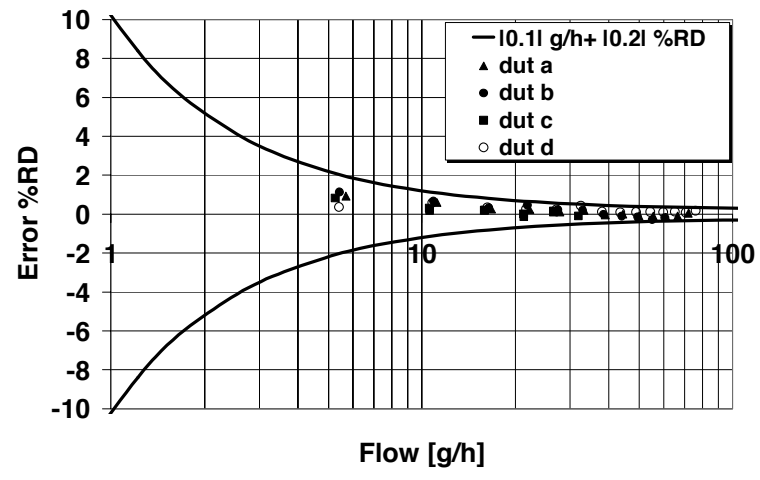

Chart 1b Accuracy of M12 (CO2)

\subsection{Zero stability vs. temperature}

The outcome of zero stability vs. temperature is depicted below. Chart $2 a$ shows the temperature-in-time profile during the experiment. As the climate chamber jumps to various temperatures the temperature sensor inside the instruments (dut1\&2) measure the more gradual heating and cooling inside the instrument. The zero as function of temperature shows reproducible behavior as depicted in chart $3 \mathrm{~b}$. This chart shows all measured points of the zero at all temperatures. The zero shows a minor drift due to temperature. This temperature drift (derivative of chart 3a) is shown in chart 2c.

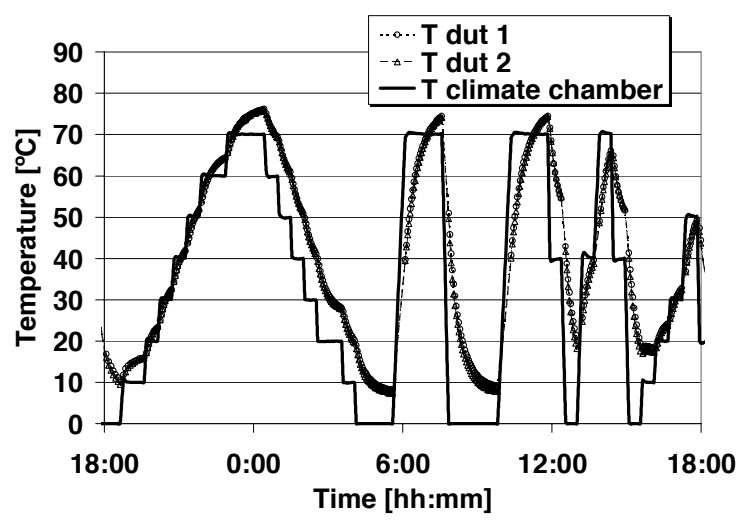

Chart 2a Temperature during M12 zero stability test 


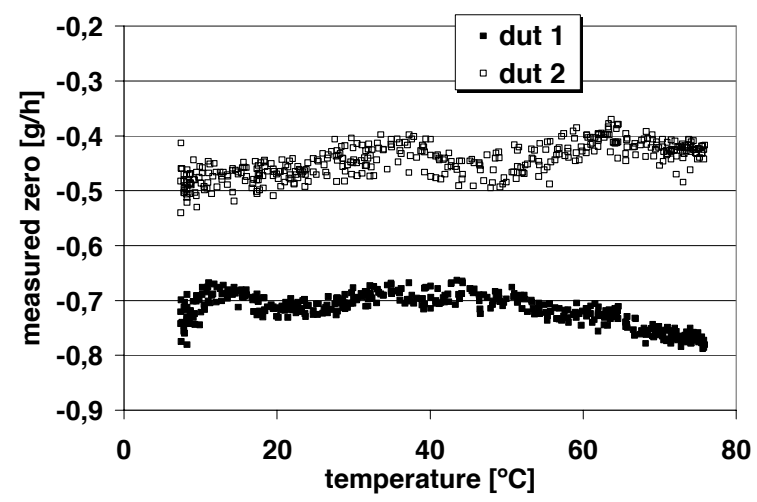

Chart $2 b$ Zero stability of M12 vs. temperature

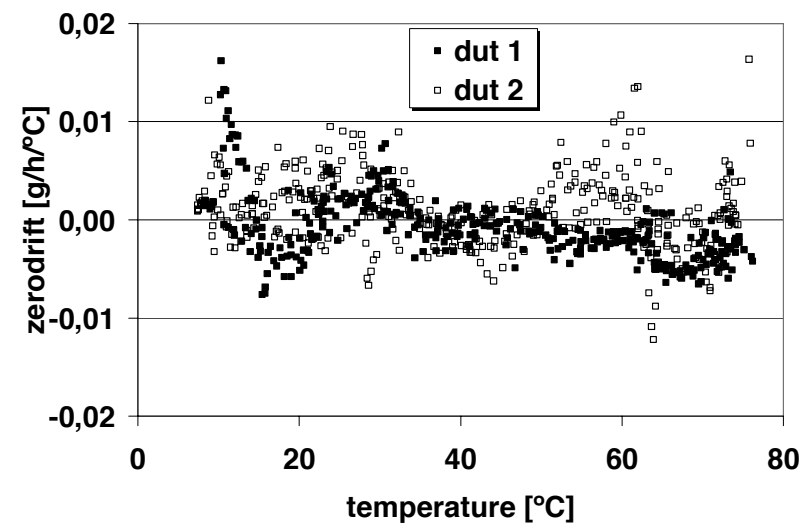

Chart 2c M12 Zero drift due to temperature

The temperature drift of the zero shows a reproducible and very small effect. Two times the standard deviation (95\% of the values) of the data in chart $3 \mathrm{c}$ results in a zero drift due to temperature of \pm 7.5 $\mathrm{mg} / \mathrm{h} /{ }^{\circ} \mathrm{C}$. This makes the instrument suitable for measuring flow accurately at changing process temperature conditions.

\subsection{Pressure drop}

The charts below show the pressure drop of the M12 instruments for air and water. Besides the pressure drop over the meter the pressure drop over a controller is also shown for the largest orifice possible in combination with the M12 meter.

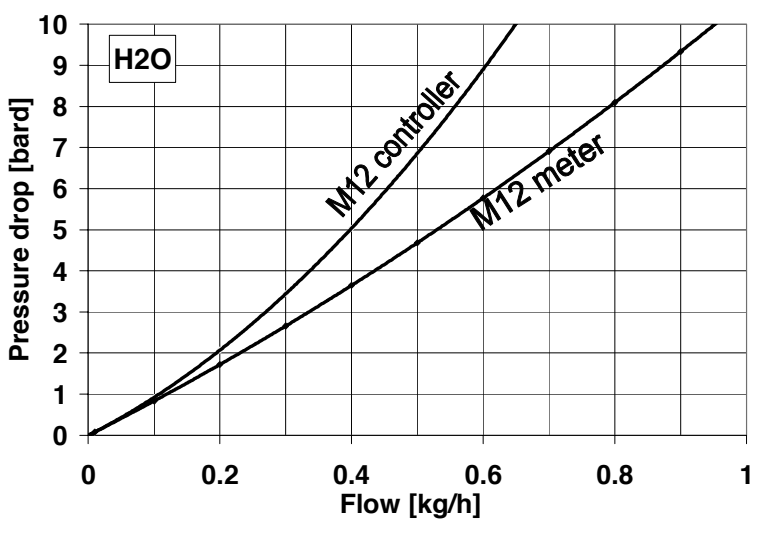

Chart 4 Pressure drop M12 air (P2 = 1bara)

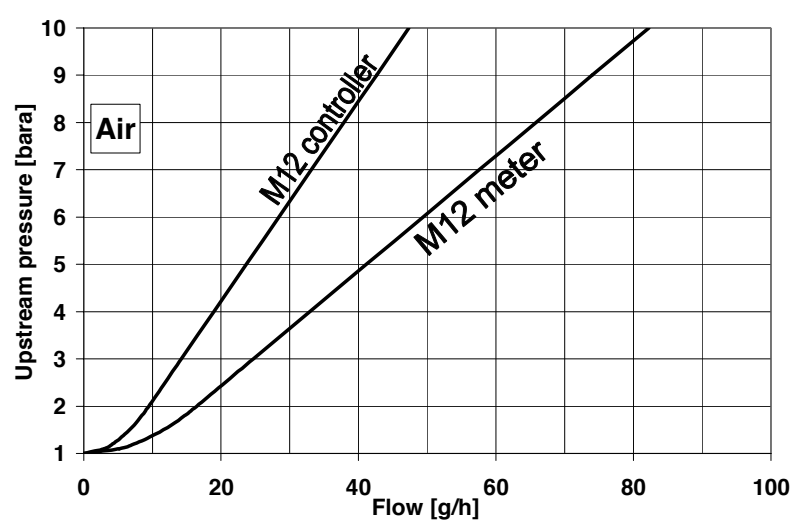

Chart 4b Pressure drop M12 water

An example from these charts is that the required inlet pressure for $20 \mathrm{~g} / \mathrm{h}$ (or $250 \mathrm{ml} / \mathrm{min}$ ) of air for a $\mathrm{M} 12$ meter equals 1.4 barg. For a controller the required inlet pressure should be at least 3 barg to get $20 \mathrm{~g} / \mathrm{h}$ of air. For measuring $60 \mathrm{~g} / \mathrm{h}$ (or $1 \mathrm{ml} / \mathrm{min}$ ) of water, an $\mathrm{M} 12$ meter requires at least $500 \mathrm{mbard}$. 


\section{Conclusions}

A mass flow meter for measuring low flow rates using the Coriolis principle has been designed, build and tested. The discussed instrument is commercially available at the moment. The design shows that optimization on tube shape, actuation and sensing principle contribute to an instrument that performs very good. Experiments have shown that the accuracy and zero stability result in a product that is the only of its kind currently available. This makes the product ideal for measuring low flow rates of liquids and gasses.

The M12 instrument (lowest flow range) is tested for accuracy on water and $\mathrm{CO}$. On both media the instrument performs very well with an accuracy of better than $\pm 0.2 \%$ reading $\pm 0.1 \mathrm{~g} / \mathrm{h}$ at all checked points.

The zero stability versus temperature shows excellent performance for the $\mathrm{M} 12$ of $\pm 7.5 \mathrm{mg} / \mathrm{h} /{ }^{\circ} \mathrm{C}$. At the lowest full scale range of $20 \mathrm{~g} / \mathrm{h}$ this results in an error of $0.0375 \% /{ }^{\circ} \mathrm{C}$ full scale which is very good.

Remarkable is that during the temperature test the climate chamber 'jumps' between extreme temperatures. Even under these circumstances the instruments zero follows the same path as if temperature would change gradually.

The pressure drop over a meter and controller is made clear by measurement. Pressure drop charts can be used to match an instrument to the application conditions.

\section{References}

[1] Mass flow meter of the Coriolis type, Mehendale et al. (US Patent No,: 7,340,965)

[2] Mass flow meter of the Coriolis type, Mehendale et al. (US Patent No,: 7,353,718)

[3] Coriolis mass flow meter, Zwikker et al. (Us pat pending No US2008/0115588) 